\title{
Intravascular Ultrasound for Assessment of Residual Coarctation of the Aorta after Balloon Angioplasty in Infants
}

Mortezaeian $H^{a}{ }^{a, b}$,

Khalili Y.b,

Farrokhi M.

Tajalli S.

Mohammadi A.S. ${ }^{b}$,

Vesal A. ${ }^{b}$

Alaei F.

Firouzi A.b,

Shafe O. ${ }^{b}$,

Gohar M.F.

Qureshi S.A.

a Cardiovascular Intervention Research Center, Rajaie Cardiovascular Medical and Research Center, Iran University of Medical Sciences, Tehran, Iran

b Rajaie Cardiovascular Medical and Research Center, Iran Medical University of Medical Sciences, Valiasr Ave Niayesh Intersection, Tehran, Iran

c Nursing Care Research Center (NCRC), School of Nursing and Midwifery, Iran University of Medical Sciences, Tehran, Iran

d Department of Pediatric Cardiology, Faculty of Medicine, Mofid Children's Hospital, Shahid Beheshti University of Medical Sciences, Tehran, Iran

e Children Growth Research Center, Research Institute for Prevention of Non-Communicable Diseases, Qazvin University of Medical Sciences, Qazvin, Iran

f Evelina London Children's Hospital, London, United Kingdom

\section{Abstract}

Intravascular ultrasound (IVUS) has been introduced as an accurate and minimally invasive diagnostic technique for the assessment of vascular anatomy and its abnormalities. We believe that IVUS can be used for clarifying the reasons for failure of balloon angiography in infantile coarctation of the aorta (CoA), because post-balloon angioplasty tearing, intimal flap, thrombosis and pseudoaneurysm of the aorta can be evaluated by IVUS with greater sensitivity and specificity. We aimed to assess the outcome of balloon angioplasty of CoA using angiography as the gold standard and IVUS as a new method in infants, comparing the two techniques for the evaluation of the diameter and area of CoA segment pre- and post-procedure. This cross-sectional study was performed on 18 infants hospitalized with a final diagnosis of CoA. All the infants underwent 
angiography and were also assessed by IVUS to measure the preoperative and postoperative diameter of the narrow segment in the two anterior-posterior and lateral views. In assessment by IVUS, the mean diameter of the coarctation site increased from $2.10 \pm 0.30 \mathrm{~mm}$ to $4.50 \pm 0.94 \mathrm{~mm}$ $(\mathrm{P}<0.001)$. Similarly, the average minimum area of the coarctation level increased from $5.26 \pm$ $1.50 \mathrm{~mm}^{2}$ to $13.77 \pm 3.48 \mathrm{~mm}^{2}$ after angioplasty $(\mathrm{P}<0.001)$. Comparing these findings, angiography and IVUS showed a high level of agreement. In the assessment of a dissection flap, there was a high level of agreement between angioplasty and IVUS before the procedure, but IVUS had higher accuracy after the procedure. Our study showed that IVUS was more reliable than angiography in the assessment of residual coarctation. IVUS yielded high sensitivity (58.3\%) and specificity (100\%) for discriminating the presence and absence of residual coarctation as well as the need for repeating the procedure. The assessment of coarctation before and after angioplasty procedures in children is possible using the IVUS method, with high accuracy. IVUS can offer greater accuracy than angiography in the evaluation of the coarctation area, detecting tears, dissection and flaps, and assessment of residual coarctation. (C) 2021, Springer Science+Business Media, LLC, part of Springer Nature.

\section{Author keywords}

Angiography; Aortic coarctation; Child; Ultrasonography 